\title{
Structural, Optical and Electrical Properties of Eu-doped CuS Nanoparticles Synthesized Through the Aqueous Route
}

\author{
Nouha Loudhaief, Mohamed Ben Salem*, Mouldi Zouaoui \\ Faculty of Sciences of Bizerte LR01ES15, University of Carthage, Bizerte, Tunisia \\ Email address: \\ salemwiem2005@yahoo.fr (M. B. Salem) \\ ${ }^{*}$ Corresponding author \\ To cite this article: \\ Nouha Loudhaief, Mohamed Ben Salem, Mouldi Zouaoui. Structural, Optical and Electrical Properties of Eu-doped CuS Nanoparticles \\ Synthesized Through the Aqueous Route. Engineering Physics. Vol. 1, No. 4, 2020, pp. 7-14. doi: 10.11648/j.ep.20200401.12
}

Received: November 18, 2019; Accepted: November 29, 2019; Published: May 28, 2020

\begin{abstract}
Eu-doped CuS nanoparticles stabilized by L-cysteine were synthesized by a low-temperature soft aqueous route. X-Ray Diffraction (XRD) patterns of the synthesized products reveal the formation of the hexagonal structure of covellite CuS. Scanning Electron Microscopy (SEM) images depict that the as-prepared nanoparticles exhibit relatively sphere like shaped morphology. Transmission Electron Microscopy (TEM) analyses show that the average size of the nanoparticles was found to be reduced with increasing the Eu concentration. UV-Visible optical absorption measurements reveal that the optical band gap is increased with increasing the Eu concentration, showing the presence of a blue shift due to quantum size effects. Impedance spectra were well modelled by introducing an electrical equivalent circuit. The electrical conductivity was found to be increased with increasing the Eu concentration. The temperature dependence of the DC conductivity confirmed the semiconducting nature of the as-prepared nanoparticles and was found to obey the Arrhenius law with two activation energies. The frequency dependence of the AC conductivity has been analyzed by Jonscher's power law suggesting the non-overlapping small polaron tunneling (NSPT) type of conduction. The polaron hopping energy was found to be increased with increasing the $\mathrm{Eu}$ concentration. The dielectric constant of the as-synthesized nanoparticles was found to be decreased with the increase in Eu concentration. The dielectric loss tangent was found to be decreased and then increased at higher frequencies with increasing the Eu concentration.
\end{abstract}

Keywords: Nanoparticles, Chemical Synthesis, Optical Properties, Electrical Properties, Dielectric Response

\section{Introduction}

Semiconductor nanostructures have attracted much interest in the scientific community because of their unique characteristics that cannot be obtained from conventional bulk materials. Owing to their unique size-dependent properties, quantum confinement, and large surface to volume ration of atoms [1], semiconductor nanostructures display novel structural, optical, electronic and magnetic properties, and because of which they find many important technological applications. Currently, more attention is paid to the synthesis and characterization of transition metal chalcogenide compounds at nanoscale because of their novel physical and chemical properties and extensive applications. Among the different chalcogenides, covellite copper sulfide
$(\mathrm{CuS})$ is extensively studied in past few years due to their versatility, availability and low-toxic nature, making its use in wide range of applications from energy to biomedical field. $\mathrm{CuS}$ is a semiconductor with direct band gap. The copper vacancies present in the $\mathrm{CuS}$ act as electron acceptors, making it a p-type semiconductor [2]. it can also be transformed into superconductor at about $1.6 \mathrm{~K} \mathrm{[3].} \mathrm{CuS}$ is a promising material with potential applications in many fields such as optoelectronic devices [4], photocatalysis [5], photovoltaic cells [6], sensors [7], batteries electrodes [8] and tissue imaging [9]. Furthermore, $\mathrm{CuS}$ nanostructures with various morphologies, such as nanoparticles [10], nanowires [11], nanorods [12], nanoplates [13], nanoribbons [14], and 
nanotubes [15] have been synthesized over the years with several methods including chemical co-precipitation method [10], hydrothermal method [16], solvothermal method [17], combustion method [18] and solid state reaction method [19].

Many efforts have been made to develop nanostructures with superior optical and electrical characteristics. Incorporation of rare-earth ions into chalcogenide semiconductors has been the subject of numerous investigations [20]. Generally, rare earth elements are incorporated in semiconductors to improve the luminescence efficiency by creating new electron energy level or perturbing the host energy level and Europium (Eu) is a suitable dopant since it is one of the most reactive rare earth elements.

Herein, a low-temperature soft aqueous route was employed to synthesize $\mathrm{Cu}_{1-\mathrm{x}} \mathrm{Eu}_{\mathrm{x}} \mathrm{S}(\mathrm{x}=0.00,0.01,0.03,0.05)$ nanoparticles. Besides, the structure, composition, morphology, particle size, optical properties and electrical properties were analyzed.

\section{Experimental Details}

\subsection{Synthesis of Eu-doped CuS Nanoparticles}

Undoped and 1\%, 3\%, and 5\% Eu-doped $\mathrm{CuS}$ nanoparticles stabilized by L-cysteine were prepared by aqueous method. Copper (II) sulfate pentahydrate $\left(\mathrm{CuSO}_{4} .5 \mathrm{H}_{2} \mathrm{O}\right.$, min. 99\%), europium (III) chloride hexahydrate $\left(\mathrm{EuCl}_{3} \cdot 6 \mathrm{H}_{2} \mathrm{O}, 99.9 \%\right)$ and sodium sulfide nonahydrate $\left(\mathrm{Na}_{2} \mathrm{~S} .9 \mathrm{H}_{2} \mathrm{O}, 98+\%\right)$ were used as precursors and L-cysteine $\left(\mathrm{C}_{3} \mathrm{H}_{7} \mathrm{NO}_{2} \mathrm{~S}, 97 \%\right)$ was used as surface ligand. All solutions were prepared using distilled water as the solvent. Eu-doped $\mathrm{CuS}$ nanoparticles are prepared as follows; in a two-neck round-bottomed flask, $5 \mathrm{~mL}$ of $0.1 \mathrm{M}$ $\mathrm{CuSO}_{4} .5 \mathrm{H}_{2} \mathrm{O}$, x $\mu \mathrm{L}$ of $0.1 \mathrm{M} \mathrm{EuCl}_{3} \cdot 6 \mathrm{H}_{2} \mathrm{O}$ and $20 \mathrm{~mL}$ of 0.1 $\mathrm{M} \mathrm{C}_{3} \mathrm{H}_{7} \mathrm{NO}_{2} \mathrm{~S}$ were combined. The $\mathrm{pH}$ of this solution was adjusted to 11 using $2 \mathrm{M}$ sodium hydroxide $(\mathrm{NaOH}, \min$. $99 \%$ ). The solution was then degassed by bubbling argon gas for 30 min after which $4.5 \mathrm{~mL}$ of degassed $0.1 \mathrm{M} \mathrm{Na}_{2} \mathrm{~S}_{2} 9 \mathrm{H}_{2} \mathrm{O}$ were quickly injected into the solution. The reaction mixture was then brought to reflux and stirred at $95{ }^{\circ} \mathrm{C}$. After $4 \mathrm{~h}$ of heating, the solution was cooled down to room temperature. The obtained precipitates were collected, washed with ethanol for several times and then dried at $60^{\circ} \mathrm{C}$ in air before further characterization.

\subsection{Characterization}

The as-synthesized Eu-doped $\mathrm{CuS}$ nanoparticles were characterized using various analytical techniques. Identity, structure, purity and crystallite size were estimated by X-ray powder diffraction (XRD) using a Bruker D8 advance X-ray diffractometer with $\mathrm{CuK}_{\alpha}$ radiations $(\lambda=1.5418 \AA)$. The morphology was scrutinized using a JEOL-JEM 5510 scanning electron microscopy (SEM). The average particle size and the phase composition were examined using a FEI Tecnai G2 transmission electron microscopy (TEM) coupled with energy dispersive X-ray spectroscopy (EDXS) system by placing a drop of the particles in ethanol onto a carbon film supported gold grid. The optical absorption measurements were carried out using a T60 UV-Visible spectrophotometer. The photoluminescence (PL) properties were measured using a Perkin Elmer LS-55 Fluorescence Spectrometer at room temperature. Electrical properties were performed using a Hewlett-Packard HP 4192A Impedance Analyzer in a frequency range $(10 \mathrm{~Hz}-3 \mathrm{MHz})$ at various temperature $\left(30^{\circ} \mathrm{C}-130^{\circ} \mathrm{C}\right)$.

\section{Results and Discussion}

\subsection{Structural Analysis}

Figure 1(a) shows XRD patterns of the $\mathrm{Cu}_{1-\mathrm{x}} \mathrm{Eu}_{\mathrm{x}} \mathrm{S}(\mathrm{x}=0.00$, $0.01,0.03,0.05)$ nanoparticles at room temperature. It can be noted that the diffraction peaks of all samples could be indexed to the hexagonal covellite phase with space group $\mathrm{P}_{3} / \mathrm{mmc}$. The average crystallite size (D) was estimated from the Debye-Scherer formula,

$$
D=\frac{k \lambda}{\beta \cos \theta}
$$

where the constant $k$ is the shape factor $=0.89, \lambda$ is the wavelength of $\mathrm{X}$-ray radiation and $\beta$ is the full width at half maximum of the peak at diffraction angle $\theta$. The average crystallite sizes of $\mathrm{Cu}_{1-\mathrm{x}} \mathrm{Eu}_{\mathrm{x}} \mathrm{S}$ nanoparticles are given in table 1 and found to be reduced with increasing the $\mathrm{Eu}$ dopant concentration. Furthermore, the lattice parameters for the samples were analyzed by the Rietveld refinement method (Table 1). It may noticed that $a$ and $c$ increase with increasing the Eu dopant concentration.

\subsection{Morphological and Microstructural Studies}

Energy dispersive X-ray spectroscopy (EDXS) studies of the Eu-doped $\mathrm{CuS}$ nanoparticles indicate the presence of copper, sulfur and europium elements with the expected stoichiometric ratios (Table 1). All the samples exhibit the same morphology, as an example, we give in figure 1(b) and figure $1(\mathrm{c})$ the scanning electron microscopic image of $\mathrm{Cu}_{1 \text { - }}$ ${ }_{x} \mathrm{Eu}_{\mathrm{x}} \mathrm{S}(\mathrm{x}=0.05)$ and the transmission electron microscopic image of $\mathrm{Cu}_{1-\mathrm{x}} \mathrm{Eu}_{\mathrm{x}} \mathrm{S} \quad(\mathrm{x}=0.01)$, repectively. SEM image depicts that the as-prepared nanoparticles exhibit relatively sphere like shaped morphology with random aggregation of tiny crystalline nuclei (Figure 1(b)). TEM image shows that particles have a spherical morphology (Figure 1(c)). Based on statistical analysis of numerous entities in many regions of all samples, the average size of the nanoparticles was shown in table 1 and was found to be reduced with increasing the Eu dopant concentration, which is in good agreement with the results estimated from the XRD technique. 
Table 1. Size, lattice parameters, optical band gap and elemental composition of $C u_{1-x} E u_{x} S$ nanoparticles.

\begin{tabular}{|c|c|c|c|c|c|c|c|}
\hline \multirow[b]{2}{*}{$\mathbf{x}$} & \multicolumn{2}{|c|}{ Size (nm) } & \multicolumn{2}{|c|}{ Lattice parameters } & \multirow[t]{2}{*}{ Optical band gap $E_{g}(\mathrm{eV})$} & \multicolumn{2}{|c|}{ Measured elements (\% Atomic) } \\
\hline & XRD & TEM & $\boldsymbol{a}(\AA)$ & $\boldsymbol{c}(\AA)$ & & $\mathbf{C u}$ & Eu \\
\hline 0.00 & 13.3 & 11.2 & 3.7982 & 16.3730 & 2.39 & 100.0 & 0.0 \\
\hline 0.01 & 12.8 & 10.5 & 3.7986 & 16.3923 & 2.43 & 98.7 & 1.3 \\
\hline 0.03 & 12.1 & 9.9 & 3.7996 & 16.4257 & 2.49 & 97.4 & 2.6 \\
\hline 0.05 & 11.6 & 9.3 & 3.8006 & 16.4578 & 2.54 & 94.9 & 5.1 \\
\hline
\end{tabular}
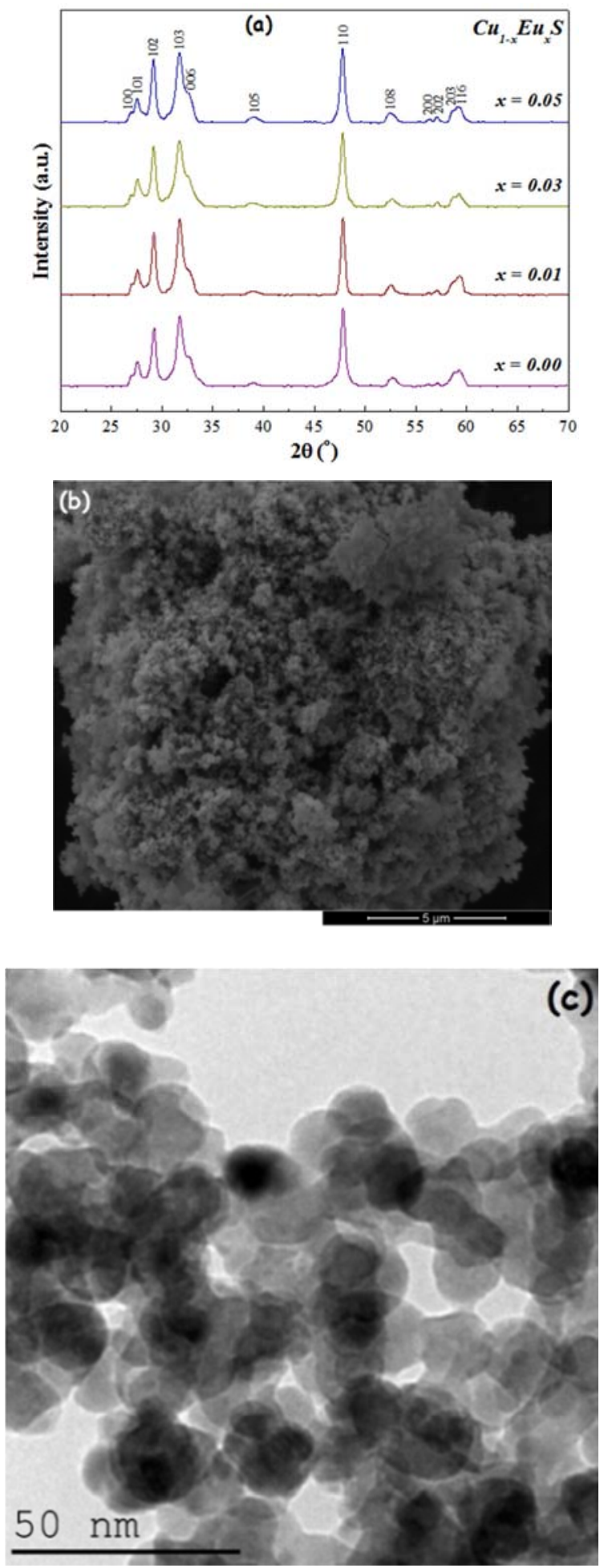

Figure 1. (a) X-Ray powder diffraction patterns of $C u_{1-x} E u_{x} S$ nanoparticles, (b) SEM image of $C u_{1-x} E u_{x} S(x=0.05)$ and (c) TEM image of $C u_{1-x} E u_{x} S$ nanoparticles $(x=0.01)$.

\subsection{Optical Absorption Studies}

The UV-Visible optical absorption spectra of $\mathrm{Cu}_{1-\mathrm{x}} \mathrm{Eu}_{\mathrm{x}} \mathrm{S}$ nanoparticles $(\mathrm{x}=0.00,0.01,0.03,0.05)$ is shown in figure 2(a). It is clear that the as-synthesized Eu-doped $\mathrm{CuS}$ nanoparticles exhibit strong photo-absorption in the wavelength between $300 \mathrm{~nm}$ and $600 \mathrm{~nm}$. In addition, all the samples show increased absorption in the near-IR region which are ascribed to the absorption of covellite CuS phase [21]. Further, the absorption edge shifts towards the lower wavelength with increasing the Eu dopant concentration; this indicates the decrease in particle size, as obtained from XRD and TEM. The energy band gap $E_{g}$ can be calculated using the relation below,

$$
\alpha h v=A\left(h v-E_{g}\right)^{n}
$$

where $\alpha$ is the absorption coefficient, $h$ is the Planck constant, $v$ is the frequency of the incident photon energy, $A$ is a constant and $n$ depends on the type of transition and is equal to $\frac{1}{2}$ for a direct band gap semiconductor. The energy band gaps $E_{g}$ of $\mathrm{Cu}_{1-\mathrm{x}} \mathrm{Eu}_{\mathrm{x}} \mathrm{S}$ nanoparticles were determined by Tauc's plot. The estimated band gap value of undoped $\mathrm{CuS}$ nanoparticles is $2.39 \mathrm{eV}$, which is higher than the band gap of $\mathrm{CuS}$ at bulk counterpart $(2.20 \mathrm{eV})[21]$ and it is the direct evidence of quantum confinement associated with nanoscale regime of the particles. The measured band gap values of $\mathrm{Cu}_{1-\mathrm{x}} \mathrm{Eu}_{\mathrm{x}} \mathrm{S}(\mathrm{x}=0.01,0.03,0.05)$ nanoparticles are $2.43 \mathrm{eV}$, $2.49 \mathrm{eV}$ and $2.54 \mathrm{eV}$, respectively, as listed in table 1 . As can be seen, $E_{g}$ is increased with increasing the Eu dopant concentration, showing the blue shift which can be explained by the confinement of electrons and holes in extremely small volume of space and formation of smaller nanosized as dopant concentration increased. This effect is also known as the Burstein-Moss shift [22].

\subsection{Photoluminescence Studies}

The PL spectra at room temperature excited with a wavelength of $360 \mathrm{~nm}$ for $\mathrm{Cu}_{1-\mathrm{x}} \mathrm{Eu}_{\mathrm{x}} \mathrm{S}(\mathrm{x}=0.00,0.01,0.03$, $0.05)$ nanoparticles are shown in figure 2(b). It is clear that all samples exhibit two blue emission bands located at 460$470 \mathrm{~nm}$ and 480-483 nm, two green emission bands situated at $519-525 \mathrm{~nm}$ and $547-550 \mathrm{~nm}$, as well as an orange emission hump positioned at $600 \mathrm{~nm}$. This peak at $600 \mathrm{~nm}$ is attributed to the energy level of the defect since its intensity is sensitive to the concentration of $\mathrm{Eu}^{3+}$ in the $\mathrm{CuS}$ crystal lattice and becomes sharper as increasing Eu concentration, which is the result of the ${ }^{5} D_{0}-{ }^{7} F_{1}$ transition of the $\mathrm{Eu}^{3+}$ ions [23]. The observed PL peaks are markedly blue shifted with increasing Eu concentration. This systematic shift of the emission bands towards the lower wavelength is due to the 
decrease of the particle size, indicating the quantum confinement effect. From figure 2(b), it obvious that the luminescence intensity increases with increasing $\mathrm{Eu}$ concentration due to the higher recombination rate of electrons and holes [24].

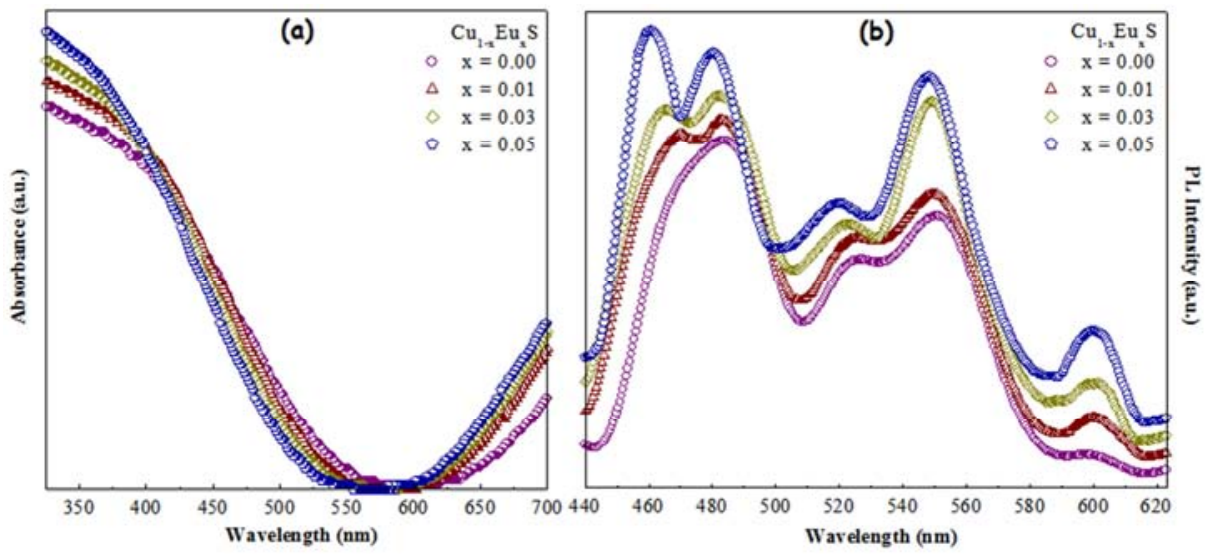

Figure 2. (a) Optical absorption spectra and (b) room temperature photoluminescence spectra of $C u_{1-x} E u_{x} S$ nanoparticles.

\subsection{Electrical Properties}

\subsubsection{Impedance Spectroscopy Analysis}

The complex impedance is given by,

$$
Z^{*}=Z^{\prime}-j Z^{\prime \prime}
$$

where $Z^{\prime}$ is the real part of impedance and $Z^{\prime \prime}$ is the imaginary part of impedance. Figure 3 shows impedance spectra of $\mathrm{Cu}_{1-\mathrm{x}} \mathrm{Eu}_{\mathrm{x}} \mathrm{S}(\mathrm{x}=0.00,0.01,0.03,0.05)$ nanoparticles, measured at various temperatures from 30 to $130{ }^{\circ} \mathrm{C}$. The inset of figure 3 shows the simple equivalent circuit comprising a parallel $R_{p}-C P E\left(Q_{p}\right)$ network serially connected to $R_{s}$; where $R_{s}$ is the series resistance which may be arises due to the electrode effect, $R_{p}$ is the DC resistance and $C P E\left(Q_{p}\right)$ (Constant Phase Element) is introduced in the circuit corresponding to the grain boundary properties due to non-ideal capacitive behavior [25].

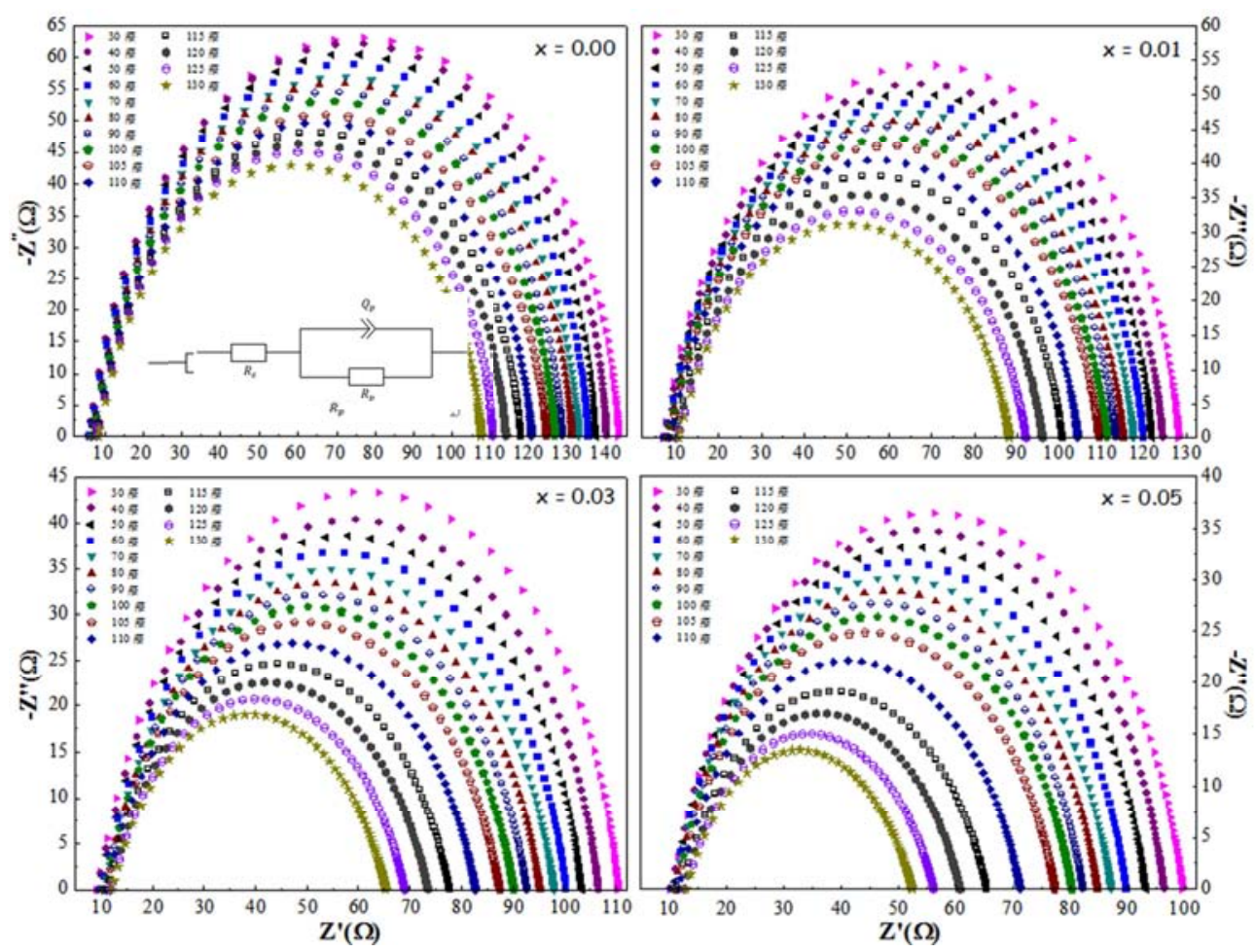

Figure 3. Nyquist plots of $\mathrm{Cu}_{1-x} E u_{x} S$ nanoparticles. Inset: Equivalent circuit.

The capacitance values $C_{p}$ for all samples at all temperatures are calculated using relation given below [25],

$$
C_{p}=\left(Q_{p} \cdot R_{p}^{1-\alpha}\right)^{\frac{1}{\alpha}}
$$

where, $\alpha$ is the degree of deviation $(0<\alpha<1)$; $\alpha=1$ for pure capacitor and $\alpha=0$ for pure resistor. The experimental complex impedance data were fitted by the EC-Lab fitting software. The variation of the fitted values, $R_{p}$ and $C_{p}$, with 
temperature for all the samples are shown in figure 4(a-b). The decrease of $R_{p}$ and the increase of $C_{p}$ with increasing in

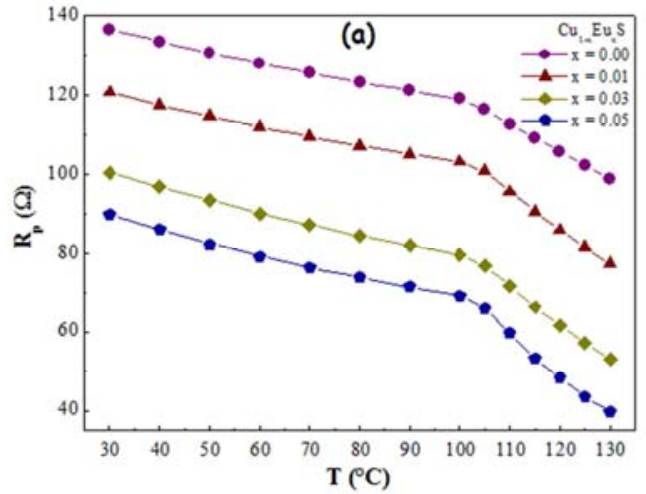

temperature indicate the semiconducting nature of samples.

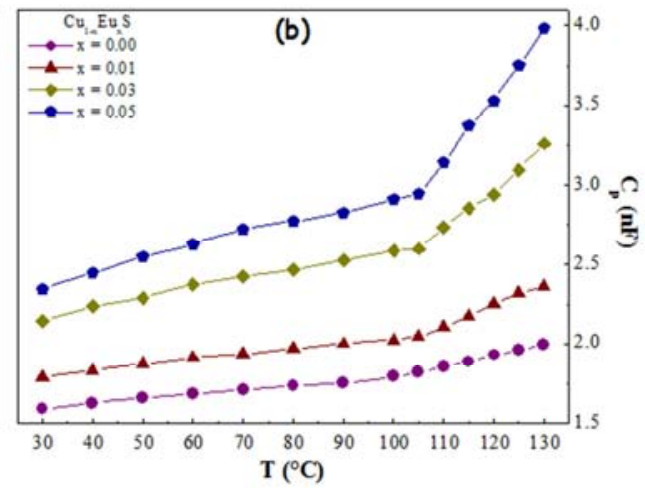

Figure 4. Variation of (a) resistance $R_{p}$ and (b) capacitance $C_{p}$ values with temperature for $C u_{1-x} E u_{x} S$ nanoparticles.
All samples exhibit similar behavior of variation of imaginary part of impedance versus logarithmic angular frequency. For example, figure 5(a) shows $Z^{\prime \prime}$ as a function of $\log (\omega)$ at different temperatures of $\mathrm{Cu}_{1-\mathrm{x}} \mathrm{Eu}_{\mathrm{x}} \mathrm{S}(\mathrm{x}=0.05)$ nanoparticles. With the rise in temperature, the intensity of the peaks of all the samples gradually decreases because the impedance values are reducing with the increase in temperature. Furthermore, as shown in the inset of figure 5(a), the peaks of $Z^{\prime \prime}$ shifts towards higher frequency as the temperature increases which implies that the rate of hopping of localized charge carrier increases [25]. Moreover, the asymmetric broadening of peaks indicates the non-Debye relaxation involved with electrical processes in the material [26].

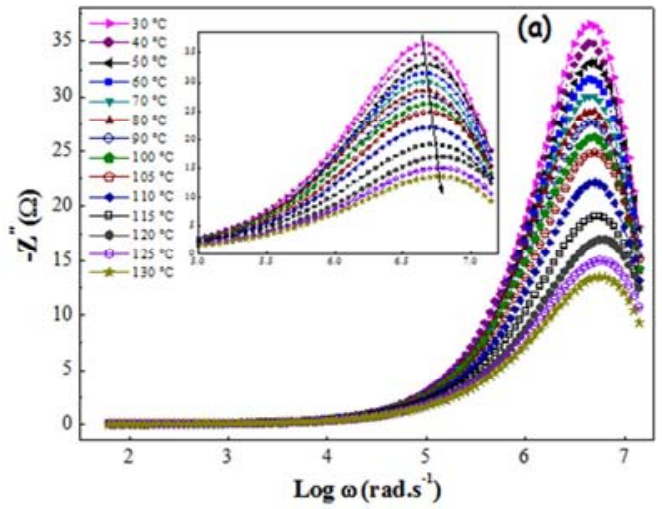

\subsubsection{Electrical Conductivity}

The electrical conductivity $(\sigma)$ can be calculated using expression given as follows [27],

$$
\sigma=\frac{t}{s} \frac{Z^{\prime}}{Z^{\prime 2}+Z^{\prime \prime 2}}
$$

where $t$ and $s$ are the thickness and the cross sectional area of the samples. We note that all samples have similar behavior of variation of logarithmic electrical conductivity versus logarithmic angular frequency. For instance, figure 5(b) shows $\log (\sigma)$ as a function of $\log (\omega)$ at various temperatures of $\mathrm{Cu}_{1-\mathrm{x}} \mathrm{Eu}_{\mathrm{x}} \mathrm{S}(\mathrm{x}=0.05)$ nanoparticles.

Figure 5. Variation of (a) imaginary part of impedance $\left(Z^{\prime \prime}\right)$ and (b) electrical conductivity $(\sigma)$ with angular frequency at different temperatures for $C u_{1-x} E u_{x} S$ $(x=0.05)$ nanoparticles.

It is observed that the electrical conductivity $(\sigma)$ presents two distinct regions within the angular frequency, corresponding to different conduction mechanism; the plateau region (DC conductivity $\left(\sigma_{D C}\right)$ ) and the dispersive region (AC conductivity $\left(\sigma_{A C}\right)$ ). The frequency dependence of electrical conductivity $(\sigma)$ can be described through Jonscher's power law [28] and it is defined as,

$$
\sigma=\sigma_{D C}+\sigma_{A C} \text { with } \sigma_{A C}=A \omega^{m}
$$

where $A$ is the proportionality constant and $m$ is the frequency exponent which is used to understand the conduction mechanism in materials. The conductivity $\sigma_{D C}$ for $\mathrm{Cu}_{1-\mathrm{x}} \mathrm{Eu}_{\mathrm{x}} \mathrm{S}$ nanoparticles can be estimated from figure 5(b). It can also be calculated using the relation, $\sigma_{D C}=\frac{t}{s R_{p}} \cdot \sigma_{D C}$ versus $\frac{1}{T}$ of $\mathrm{Cu}_{1-\mathrm{x}} \mathrm{Eu}_{\mathrm{x}} \mathrm{S}$ nanoparticles are shown in figure 6(a). The DC conductivity is found to be increased with the increase in temperature; this indicates that the electrical conductivity mechanism may be thermally activated process. The relation between DC conductivity $\left(\sigma_{D C}\right)$ and temperature $(T)$ follows Arrhenius law given as follows, 


$$
\sigma_{D C}=\sigma_{0} e^{-\frac{E_{a}}{k_{B} T}}
$$

where $\sigma_{0}$ is the pre-exponential factor, $E_{a}$ is the activation energy and $k_{B}$ is the Boltzmann constant. As can be seen from figure 6(a), Arrhenius plots are consisting by two parts referred as part $\mathrm{I}\left(T \leq 100^{\circ} \mathrm{C}\right)$ and part $\mathrm{II}\left(T>100^{\circ} \mathrm{C}\right)$, implies, two activation energies, $E_{a 1}$ (part I) and $E_{a 2}$ (part II). The evaluated values of the activation energies are summarized in table 2. It is clear that in both parts, the activation energies are found to be increased with the increase of Eu concentration. In the dispersive region, the parameters $A$ and $m$ of $\sigma_{A C}$ can be obtained by fitting the plot of $\log (\sigma)$ versus $\log (\omega)$. In our case and regarding the variation of $m$ versus temperature for $\mathrm{Cu}_{1-\mathrm{x}} \mathrm{Eu}_{\mathrm{x}} \mathrm{S}$ nanoparticles, it is found that the exponent $m$ remains less than a unity and increases with the increase in temperature for all the samples. So, the non-overlapping small polaron tunneling (NSPT) [27, 29] seems to be the most model related to the obtained results. In this model, small polarons are formed when a charge carrier (as moving electrons in

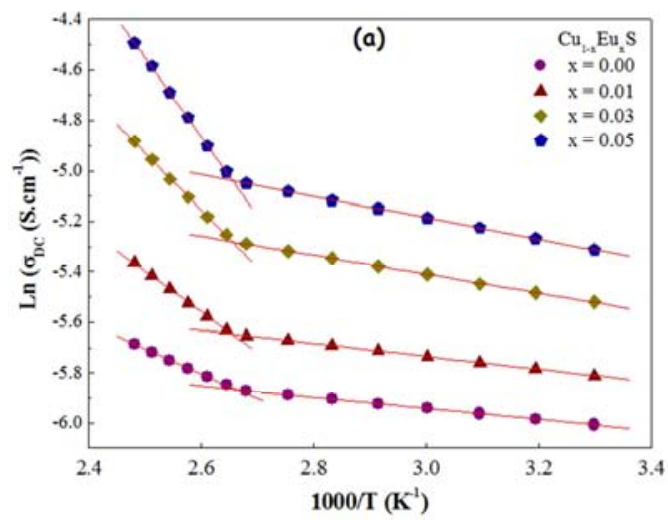

lattice) deforms the surrounding lattice, and a tunneling process is responsible for conductivity. According to this model, the frequency exponent $(m)$ is given by the following relation [29],

$$
m=1+\frac{4}{\frac{W_{m}}{k_{B} T}-\operatorname{Ln}(\omega \tau)}
$$

where $W_{m}$ is the polaron hopping energy and $\tau$ is the characteristic relaxation time that can be obtained from the value of $\omega_{0}$ corresponds to the maximum of the peak of $Z^{\prime \prime}$ versus $\log (\omega)$ using the relation, $\tau=\frac{1}{\omega_{0}}$. In the case of $\frac{W_{m}}{k_{B} T} \gg \operatorname{Ln}(\omega \tau), m$ becomes [29],

$$
m=1+\frac{4 k_{B} T}{W_{m}}
$$

The variation of the calculated values of $W_{m}$ versus temperature is shown in figure $6(\mathrm{~b}) . W_{m}$ is found to be increased with increasing in temperature and $\mathrm{Eu}$ concentration.

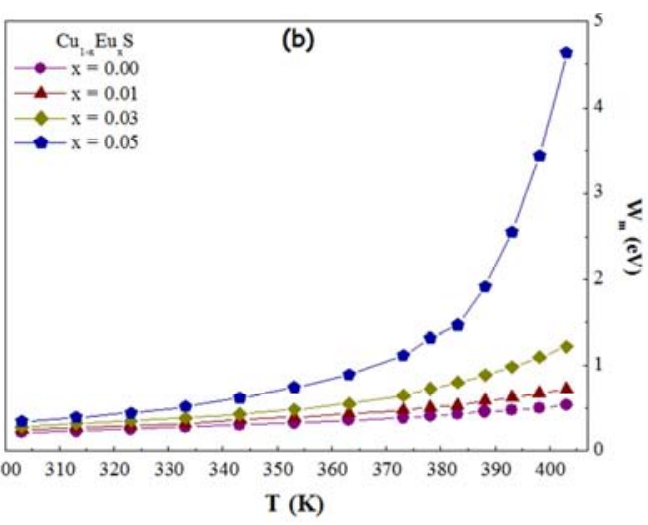

Figure 6. Variation of (a) DC conductivity $\left(\sigma_{D C}\right)$ and (b) polaron hopping energy $\left(W_{m}\right)$ with temperature for $C u_{1-x} E u_{x} S(x=0.05)$ nanoparticles.

Table 2. Activation energies of $C u_{1-x} E u_{x} S$ nanoparticles.

\begin{tabular}{lll}
\hline \multirow{2}{*}{$\mathbf{x}$} & \multicolumn{2}{c}{ Activation Energy $\left(\times \mathbf{1 0}^{-\mathbf{2}} \mathbf{e V}\right)$} \\
\cline { 2 - 3 } & $\boldsymbol{E}_{\boldsymbol{a} \mathbf{1}}$ & $\boldsymbol{E}_{\boldsymbol{a} \mathbf{2}}$ \\
\hline 0.00 & 1.90 & 8.57 \\
0.01 & 2.19 & 13.91 \\
0.03 & 3.25 & 19.51 \\
0.05 & 3.65 & 26.96 \\
\hline
\end{tabular}

\subsubsection{Dielectric Analysis}

The complex permittivity is given by,

$$
\varepsilon^{*}=\varepsilon^{\prime}-j \varepsilon^{\prime \prime}
$$

where $\varepsilon^{\prime}$ is the dielectric constant and $\varepsilon^{\prime \prime}$ is the dielectric loss. $\varepsilon^{\prime}$ and $\varepsilon^{\prime \prime}$ can be calculated using the following relations [26],

$$
\begin{aligned}
\varepsilon^{\prime} & =\frac{Z^{\prime \prime}}{\omega C_{0}\left(Z^{\prime 2}+Z^{\prime \prime 2}\right)} \\
\varepsilon^{\prime \prime} & =\frac{Z^{\prime}}{\omega C_{0}\left(Z^{\prime 2}+Z^{\prime \prime 2}\right)}
\end{aligned}
$$

where, $C_{0}=\frac{\varepsilon_{0} s}{t}$ is the free space capacitance [26] and $\varepsilon_{0}$ is the free space permittivity. The dielectric loss tangent $(\tan \delta)$ is given by the relation below,

$$
\tan \delta=\frac{\varepsilon^{\prime \prime}}{\varepsilon^{\prime}}
$$

All samples exhibit similar behavior of variation of dielectric constant versus logarithmic angular frequency. As an example, figure 7(a) shows $\varepsilon^{\prime}$ as a function of $\log (\omega)$ of $\mathrm{Cu}_{1-\mathrm{x}} \mathrm{Eu}_{\mathrm{x}} \mathrm{S}(\mathrm{x}=0.05)$ nanoparticles at different temperatures. On the one hand, it is observed that $\varepsilon^{\prime}$ decreases gradually with the increase of the frequency and attains an almost constant value at higher frequencies; at low frequencies $\left(\omega \ll \omega_{0}\right), \varepsilon^{\prime}$ depends on four types of polarization (electronic, ionic, orientation and space charge polarizations) [26]. As the frequency is increased $\left(\omega<\omega_{0}\right)$, the orientation polarization is decreased and dipoles begin to lag behind the electric field, which can cause the reduction of the value of $\varepsilon^{\prime}$ with increase in frequency. When the frequency reaches the characteristic frequency $\left(\omega=\omega_{0}\right), \varepsilon^{\prime}$ drops (relaxation process). At very high frequencies $\left(\omega \gg \omega_{0}\right)$, dipoles will be completely unable to follow the field and the polarization is stopped. Thus, $\varepsilon^{\prime}$ decreased and attained a constant value [26]. On the other hand, we note that in all samples, the dielectric constant increases with increasing temperature due to the fact that, an increase in temperature facilitates more 
and more dipole to be oriented along the electric field and $\varepsilon^{\prime}$ increases also with increasing Eu dopant concentration. Further, all samples have similar behavior of variation of dielectric loss tangent versus logarithmic angular frequency. For instance, figure 7(b) shows $\tan \delta$ as a function of $\log (\omega)$ of $\mathrm{Cu}_{1-\mathrm{x}} \mathrm{Eu}_{\mathrm{x}} \mathrm{S}(\mathrm{x}=0.05)$ nanoparticles at various temperatures. All curves indicate that the dielectric loss

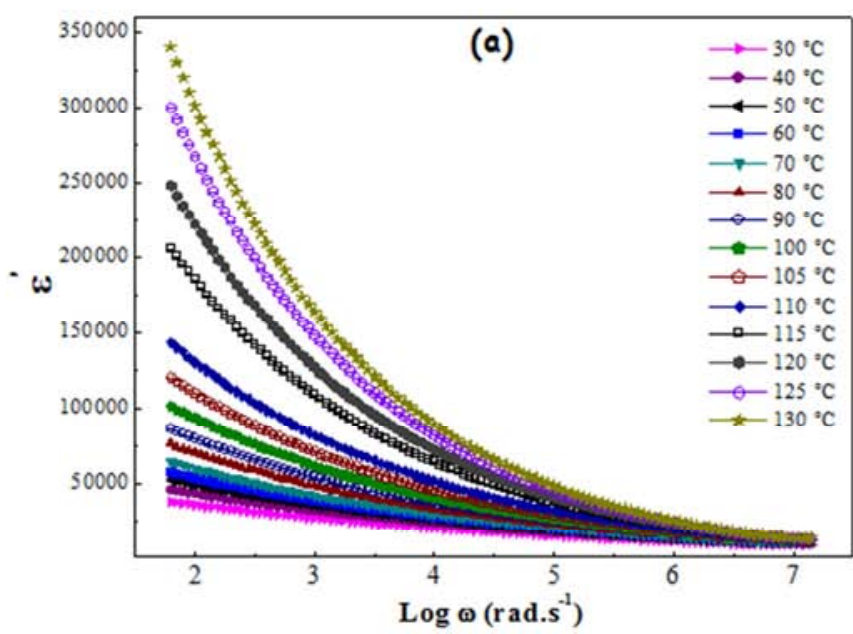

tangent is strongly dependent on frequency of the applied electric field; it decreases with increasing frequency until reaching almost a constant value. $\tan \delta$ is also strongly dependent on temperature for all the samples. This figure shows that $\tan \delta$ decreases with the increase in temperature. We note also that $\tan \delta$ decreases with increasing Eu dopant concentration.

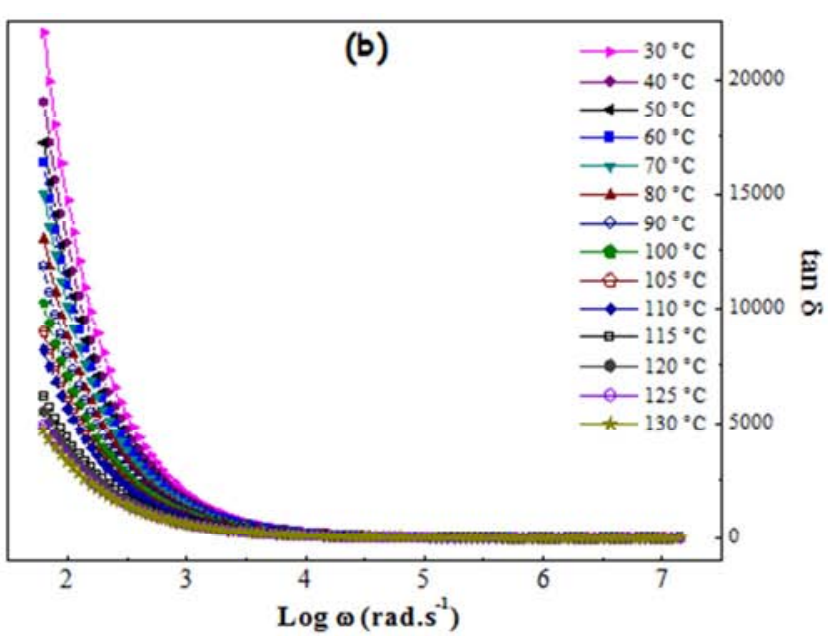

Figure 7. Variation of (a) dielectric constant $\left(\varepsilon^{\prime}\right)$ and (b) loss tangent (tan $\delta$ ) with angular frequency at different temperatures for Cu $u_{1-x}$ E $u_{x} S$ ( $x=0.05$ ) nanoparticles.

\section{Conclusion}

Low-temperature soft aqueous route was employed to synthesize Eu-doped $\mathrm{CuS}$ nanoparticles stabilized by Lcysteine. XRD analyses reveal the formation of the hexagonal structure of covellite $\mathrm{CuS}$ and indicate that the crystallite size is found to be reduced with increasing $\mathrm{Eu}$ dopant concentration. Electronic microscopy images depict that the as-prepared nanoparticles exhibit relatively sphere like shaped morphology with random aggregation and show that the average size was reduced with increasing $\mathrm{Eu}$ concentration. UV-Visible optical absorption measurements reveal that the optical band gap is increased with increasing Eu concentration, showing the presence of a blue shift due to quantum size effects. Photoluminescence measurements show a blue shift with increasing Eu concentration indicating the quantum confinement effects. The $Z^{\prime \prime}$ relaxation peaks shifted towards higher frequency with the increase in temperature and show broad asymmetric nature indicated the thermally activated relaxation and the non-Debye type. The temperature dependence of the DC conductivity was found to obey the Arrhenius law with two activation energies. The theoretical fitting of experimental data of AC conductivity suggests the non-overlapping small polaron tunneling type of conduction. Dielectric constant $\left(\varepsilon^{\prime}\right)$ of Eu-doped $\mathrm{CuS}$ nanoparticles decreases gradually with the increase in frequency and attains an almost constant value at higher frequencies. It also increases with the increase in temperature and $\mathrm{Eu}$ concentration. Dielectric loss tangent $(\tan \delta$ ) of samples was found to decrease rapidly with the increase in frequency until reaching almost a constant value. $\tan \delta$ also decreases with the increase in temperature and $\mathrm{Eu}$ concentration.

\section{References}

[1] S. Sagadevan, and J. Podder, "Investigation on structural, surface morphological and dielectric properties of $\mathrm{Zn}$-doped SnO2 nanoparticles," Mater. Res., vol. 19, pp. 420-425, 2016.

[2] J. Kundu, and D. Pradhan, "Controlled synthesis and catalytic activity of copper sulfide nanostructured assemblies with different morphologies," Mater. Interfaces, vol. 6, pp. 18231834, 2014.

[3] W. Liang, and M. H. Whangbo, "Conductivity anisotropy and structural phase transition in Covellite CuS," Solid State Commun., vol. 85, pp. 405-408, 1993.

[4] X. L. Yu, C. B. Cao, H. S. Zhu, Q. S. Li, C. L. Liu, and Q. H. Gong, "Nanometer-sized copper sulfide hollow spheres with strong optical-limiting properties," Adv. Funct. Mater., vol. 17, pp. 1397-1401, 2007.

[5] M. Tanveer, C. B. Cao, Z. Ali, I. Aslam, F. Idrees, W. S. Khan, F. K. But, M. Tahir, and N. Mahmood, "Template free synthesis of $\mathrm{CuS}$ nanosheet-based hierarchical microspheres: an efficient natural light driven photocatalyst," CrystEngComm., vol. 16, pp. 5290-5300, 2014.

[6] Y. X. Zhao, and C. Burda, "Development of plasmonic semiconductor nanomaterials with copper chalcogenides for a future with sustainable energy materials," Energy Environ. Sci., vol. 5, pp. 5564-5576, 2012.

[7] H. Lee, S. W. Yoon, E. J. Kim, and J. Park, "In-situ growth of copper sulfide nanocrystals on multiwalled carbon nanotubes and their application as novel solar cell and amperometric glucose sensor materials," Nano Lett., vol. 7, pp. 778-784, 2007. 
[8] C. H. Lai, K. W. Huang, J. H. Cheng, C. Y. Lee, B. J. Hwang, and L. J. Chen, "Direct growth of high-rate capability and high capacity copper sulfide nanowire array cathodes for lithium-ion batteries," J. Mater. Chem., vol. 20, pp. 6638-6645, 2010 .

[9] G. Ku, M. Zhou, S. Song, Q. Huang, J. Hazle, and C. Li, "Copper sulfide nanoparticles as a new class of photoacoustic contrast agent for deep tissue imaging at $1064 \mathrm{~nm}$," ACS Nano, vol. 6, pp. 7489-7496, 2012.

[10] N. Sreelekha, K. Subramanyam, D. Amaranatha Reddy, G. Murali, S. Ramu, K. Rahul Varma, and R. P. Vijayalakshmi, "Structural, optical, magnetic and photocatalytic properties of Co doped CuS diluted magnetic semiconductor nanoparticles," Appl. Surf. Sci., vol. 378, pp. 330-340, 2016.

[11] M. T. Mayer, Z. I. Simpson, S. Zhou, and D. W. Wang, "IonicDiffusion-Driven, Low-Temperature, Solid-State Reactions Observed on Copper Sulfide Nanowires," Chem. Mater., vol. 23, pp. 5045-5051, 2011.

[12] M. Kruszynska, H. Borchert, A. Bachmatiuk, M. H. Rummeli, B. Buchner, J. Parisi, and J. Kolny-Olesiak, "Size and Shape Control of Colloidal Copper (I) Sulfide Nanorods," ACS Nano, vol. 6 , pp. 5889-5896, 2012.

[13] H. B. Wu, and W. Chen, "Synthesis and reaction temperaturetailored self-assembly of copper sulfide nanoplates," Nanoscale, vol. 3, pp. 5096-5102, 2011.

[14] C. Tan, R. Lu, P. Xue, C. Bao, and Y. Zhao, "Synthesis of CuS nanoribbons templated by hydrogel," Mater. Chem. Phys., vol. 112, pp. 500-503, 2008.

[15] C. Wu, S. H. Yu, S. Chen, G. Liu, and B. Liu, "Large scale synthesis of uniform $\mathrm{CuS}$ nanotubes in ethylene glycol by a sacrificial templating method under mild conditions," J. Mater. Chem., vol. 16, pp. 3326-3331, 2006.

[16] M. Saranya, C. Santhosh, R. Ramachandran, P. Kollu, P. Saravanan, M. Vinoba, S. K. Jeong, and A. N. Grace, "Hydrothermal growth of $\mathrm{CuS}$ nanostructures and its photocatalytic properties," Powder Technol., vol. 252, pp. 2532, 2014.

[17] X. S. Hu, Y. Shen, L. H. Xu, Li. M. Wang, and Y. J. Xing, "Preparation of flower-like CuS by solvothermal method and its photodegradation and UV protection," J. Alloys Compd., vol. 674 , pp. 289-294, 2016.

[18] A. Daya Mania, M. Deepaa, P. Ghosalb, and C. Subrahmanyam, "Novel single pot synthesis of metal $(\mathrm{Pb}, \mathrm{Cu}$, Co) sulfide nanomaterials-towards a quest for paintable electrode materials that supersedes Pt electrode," Electrochim. Acta, vol. 139, pp. 365-373, 2014.
[19] W. Wang, and L. Ao, "Synthesis and characterization of crystalline $\mathrm{CuS}$ nanorods prepared via a room temperature one-step, solid-state route," Mater. Chem. Phys., vol. 109, pp. 77-81, 2008.

[20] W. Luo, Y. Liu, and X. Chen, "Lanthanide-doped semiconductor nanocrystals: electronic structures and optical properties," Sci. China Mater., vol. 58, pp. 819-850, 2015.

[21] K. Mageshwari, S. S. Mali, T. Hemalatha, R. Sathyamoorthy, and P. S. Patil, "Low temperature growth of CuS nanoparticles by reflux condensation method," Prog. Solid State Chem., vol. 39, pp. 108-113, 2011.

[22] J. W. Shin, and W. J. Cho, "Microwave Annealing Effects of Indium-Tin-Oxide Thin Films: Comparison with Conventional Annealing Methods," Phys. Status Solidi A., vol. 215, pp. $1700975,2018$.

[23] S. Horoz, B. Yakami, U. Poudyal, J. M. Pikal, W. Wang, and J. Tang, "Controlled synthesis of Eu2+ and Eu3+ doped ZnS quantum dots and their photovoltaic and magnetic properties," AIP Adv., vol. 6, pp. 045119, 2016.

[24] K. Subramanyam, N. Sreelekha, D. Amaranatha Reddy, G. Murali, K. Rahul Varma, and R. P. Vijayalakshmi, "Chemical synthesis, structural, optical, magnetic characteristics and enhanced visible light active photocatalysis of $\mathrm{Ni}$ doped $\mathrm{CuS}$ nanoparticles," Solid State Sci., vol. 65, pp. 68-78, 2017.

[25] J. H. Joshi, D. K. Kanchan, M. J. Joshi, H. O. Jethva, and K. D Parikh, "Dielectric relaxation, complex impedance and modulus spectroscopic studies of mix phase rod like cobalt sulfide nanoparticles,” Mater. Res. Bull., vol. 93, pp. 63-73, 2017.

[26] S. Sil, J. Datta, M. Das, R. Jana, S. Halder, A. Biswas, D. Sanyal, and P. P. Ray, "Bias dependent conduction and relaxation mechanism study of $\mathrm{Cu} 5 \mathrm{FeS} 4$ film and its significance in signal transport network," J. Mater. Sci. Mater. Electron., vol. 29, pp. 5014-5024, 2018.

[27] A. Rahal, S. Megdiche Borchani, K. Guidara, and M. Megdiche, "Studies of electric, dielectric, and conduction mechanism of LiNiV0.5P0.5O4," J. Alloys Compd., vol. 735, pp. 1885-1892, 2018 .

[28] S. Saha, S. Chanda, A. Dutta, and T. Sinha, "Dielectric relaxation of $\mathrm{PrFeO}_{3}$ nanoparticles," Solid State Sci., vol. 58, pp. 55-63, 2016.

[29] M. Sassi, A. Bettaibi, A. Oueslati, K. Khirouni, and M. Gargouri, "Electrical conduction mechanism and transport properties of LiCrP2O7 compound," J. Alloys Compd., vol. 649, pp. 642-648, 2015. 